\title{
Sidereal time analysis as a tool for the study of the space distribution of sources of gravitational waves
}

\author{
G. Paturel ${ }^{1}$ and Yu. V. Baryshev ${ }^{2,3}$ \\ 1 CRAL-Observatoire de Lyon, 69561 Saint-Genis Laval Cedex, France \\ 2 Astronomical Institute of the Saint-Petersburg University, 198504 St.-Petersburg, Russia \\ 3 Isaac Newton Institute of Chile, St.-Petersburg Branch, Russia
}

Received 15 July 2002 / Accepted 15 October 2002

\begin{abstract}
Gravitational wave (GW) detectors operating on a long time range can be used for the study of space distribution of sources of GW bursts or to put strong upper limits on the GW signal of a wide class of source candidates. For this purpose we propose here a sidereal time analysis to analyze the output signal of GW detectors. Using the characteristics of some existing detectors, we demonstrate the capability of the sidereal time analysis to give a clear signature of different localizations of GW sources: the Galactic Center, the Galactic Plane, the Supergalactic plane, the Great Attractor. On the contrary, a homogeneous 3D-distribution of GW sources gives a signal without features. In this paper we consider tensor gravitational waves with randomly oriented polarization. We consider GW detectors at fixed positions on the Earth, and a fixed orientation of the antenna.
\end{abstract}

Key words. gravitational waves - methods: data analysis - methods: statistical

\section{Introduction}

In this paper, we base our study of detection of gravitational waves $(\mathrm{GW})$ on a phenomenological approach as formulated by Finn (2001). We explore the reality that could emerge directly from observation, without considering specific models of GW emission. As has happened frequently in the history of astronomy when a new observational window opens, GW astronomy may lead to a revision of theoretical models and to the development of new ideas. Even upper limits on the energy of GW events are very informative because they put restrictions on the predicted characteristics of GW sources and then on the physics of GW themselves. Energetic GW bursts often arise in regions where the gravity effects are strong (supernova explosion, neutron star merging...). They thus carry information about fundamental gravity physics in an unexplored regime of strong field.

Our phenomenological approach is also justified by the fact that standard calculations of GW signals from supernova explosions and merging of relativistic compact massive objects essentially depend on the details of the last relativistic stages of the gravitational collapse that are still poorly known (Thorne 1987, 1997; Paczynsky 1999; Finn 2001).

Several GW detectors of the third generation are operating with long duty times. Five resonant $\mathrm{GW}$ detectors of the International Gravitational Event Collaboration (IGEC) already cover 1332 days of observation (Astone 2001), i.e. about

Send offprint requests to: G. Paturel, e-mail: patu@obs . univ-lyon1.fr
$90 \%$ of the total time between 1997-2000. In 2002, the laser interferometric detectors TAMA300, GEO600 and LIGO started to operate, though with shorter duty cycles.

Usual method of data analysis for GW detectors consists of searching for coincidences of events between two or more detectors (Prodi et al. 2000). Here we consider another method - sidereal time analysis (STA) - that consists of recording all GW events as a function of sidereal time. This possibility was mentioned by Weber (1969) in his pioneering work. The Earth's rotation, measured with respect to stars, gives a natural unit of time for ground-based GW detectors. The advantage of the STA method is that the noise events do not correlate with sidereal time, while true GW signals, coming from a certain distribution of GW sources, is tightly related to definite sidereal time. This happens because, for a fixed distribution of GW sources, a given antenna pattern produces a fixed amplitude shape along the sidereal time. Hence, for a given detection threshold, GW events will be detected only for specific sidereal time intervals.

Here, considering tensor waves first, we demonstrate the capability of the STA method to give a clear signature of some realistic distributions of GW sources. For this phenomenological approach we must choose a very general form of the GW burst amplitude and then a distribution of GW sources. In Sect. 2 we explain how the amplitude of individual sources is chosen and how the considered distributions of sources are built. In Sect. 3 we give, for each distribution, the relative rate of GW events along sidereal time, by considering a Weber-type bar-detector (EXPLORER) and an interferometric 
detector (VIRGO). In Sect. 4 we analyze the results to show how to use our results for a practical comparison with real observations.

Our main target is to prepare the tools for the concrete analysis of forthcoming GW signals. Our FORTRAN program is available on request.

\section{Description of normalized sources}

\subsection{Amplitude of an individual GW burst emitter}

Let us recall briefly how the count of events is made. For more details see Baryshev \& Paturel (hereafter, BP2001).

Depending on the energy transformed into GW by a source, one will observe a change in the reference length $l$. This is measured by the observed amplitude $h_{\mathrm{obs}}=\Delta l / l$. This signal will be counted as a detected event only if $h_{\text {obs }}$ is larger than the limiting amplitude $h_{\text {lim }}$ that defines the sensitivity of the detector.

Many of the expected signals may be presented as the "unit pulse of GW burst" introduced by Amaldi \& Pizzella (1979). It is a GW burst in a sinusoidal wave with amplitude $h_{0}$, frequency $v_{0}$ and duration $\tau_{\mathrm{g}}$. For tensor GW, the observed amplitude $h_{\text {obs }}$ at a distance $r$, produced by energy $E_{\mathrm{gw}}$, is (see e.g. Pizzella 1989):

$h_{\mathrm{obs}}=1.4 \times 10^{-20}\left(\frac{1 \mathrm{Mpc}}{r}\right)\left(\frac{10^{3} \mathrm{~Hz}}{v_{0}}\right)\left(\frac{E_{\mathrm{gw}}}{1 M_{\odot} c^{2}}\right)^{1 / 2}\left(\frac{1 s}{\tau_{\mathrm{g}}}\right)^{1 / 2}$.

Hence, a GW source at a fixed distance $r$, to a first approximation, is characterized by three main observable parameters $E_{\mathrm{gw}}$, $v_{0}$ and $\tau_{\mathrm{g}}$, for a wide class of radiation processes.

In the present paper, we consider the normalized amplitude that may be written as $(r$ in $\mathrm{Mpc})$ :

$$
\frac{h_{\mathrm{obs}}}{h_{\star}} \propto \frac{|G(\alpha, \delta)|}{r} \text {. }
$$

The term $G(\alpha, \delta)$ is a geometrical factor that defines the position of the source, with respect to the detector. The expression of this term is given for bar and interferometric detectors in BP2001. The quantity $h_{\star}$ defines the type of source. For galactic weak sources we will adopt $h_{\star}=10^{-20}$ or $h_{\star}=10^{-23}$. For powerful extragalactic sources of GW, like supernovae, we will adopt $h_{\star}=10^{-17}$ or $h_{\star}=10^{-19}$. Obviously, our calculations may be rescaled by using any other combinations of main GW parameters.

Because the polarization angle cannot be predicted, it has been taken at random. The net effect of this unknown polarization is that the number of events, at a certain level above the noise, is simply reduced (for more detail see BP2001). This does not change the shape of the count rate with sidereal time. Note that the polarization makes it that one cannot infer the true amplitude of a GW event from the observed amplitude. Nevertheless, a large amplitude means both a large true amplitude and a favorable polarization.

\subsection{Distribution of sources}

We now define a group of individual GW sources. The sources inside such a group have a well-defined distribution. For instance, a group can be the center of our Galaxy. In this case each individual source is a galactic emitter (like neutron star mergers or unknown sources of GW pulses) distributed in a sphere representing the region around the center of our Galaxy. Another example of a group can be a supercluster of galaxies. In this case each individual source is a galaxy. The group can be also a flat distribution of emitters with a given thickness and a given size. An example is de Vaucouleurs's Local Super Cluster (de Vaucouleurs 1956, 1975).

Each group of emitters is characterized by:

- Number of individual emitters. Generally, this number is arbitrary but, when one considers several groups, their relative populations must be fixed.

- The rate of GW burst emission, e.g. in solar masses per unit of time. Because we calculate the relative number of detected events (in percent) for an arbitrary number of sources, the rate of GW emission for each individual emitter may be set to one. This assumes that each individual emitter has the same probability of producing a GW event.

- The coordinates of each individual emitter. We adopted equatorial coordinates. So, one can use an actual catalog of emitters (like a catalog of real galaxies) or a simulated catalog, using coordinates obtained from an artificial distribution of emitters.

- Distance of each emitter. For a simulated sample the distance is given by the considered distribution. For an observed catalog of galaxies, the distance can be deduced from the radial velocity using a given Hubble constant.

There are several natural space distributions of GW sources that may be directly tested by the STA method. Here we consider the following models of GW source distributions that will be tested in the next section:

1. Homogeneous 3D-distribution. We consider a homogeneous distribution of 10000 emitters in a sphere of radius $R=10 \mathrm{kpc}$ centered on the detector. Each emitter is considered as a galactic object with a reference amplitude $h_{\star}=10^{-20}$ or $h_{\star}=10^{-22}$ for EXPLORER or VIRGO, respectively. This distribution will be used to show that, on average, there is a constant rate of GW events along sidereal time. Obviously, the result is the same when a homogeneous 3D-distribution of extragalactic objects is used (we made this verification).

2. Galactic center. The galactic center is represented by one group of 10000 emitters located at the 1950 equatorial coordinates: B174224.0-285500. The number of sources is assumed to decrease following a Gaussian distribution with standard deviation (radius) of $\sigma=0.001 \mathrm{Mpc}$. The adopted distance between the Sun and the galactic center is $0.010 \mathrm{Mpc}$. The kind of emitter is not explicitly identified, but one can imagine that the galactic center could be the source of many strong GW events. A reference amplitude $h_{\star}=10^{-20}$ is used for the kind of emitters detectable with EXPLORER and $h_{\star}=10^{-23}$ for emitters detectable with VIRGO.

3. The galactic plane. The distribution will be centered on the galactic center B174224.0-285500 at a distance of $0.010 \mathrm{Mpc}$ from the Sun. The radius of the distribution (radius of our Galaxy) is taken as $0.0125 \mathrm{Mpc}$. The adopted 
thickness of the disk is $2 \mathrm{kpc}$. We consider 10000 emitters with a reference amplitude of $h_{\star}=10^{-20}$ or $h_{\star}=10^{-23}$ for EXPLORER and VIRGO respectively. Each emitter will be distributed homogeneously in this thin disk. Note that, because our Sun is not at the center of this distribution, the distribution along the galactic longitude is not uniform. Again, for this distribution, we do not use a priori information on the types of GW sources.

4. The supergalactic plane. The dominant structure around the Local Group is de Vaucouleurs Local Super Cluster (LSC). The Virgo galaxy cluster is the dominant structure of the LSC. We will consider a homogeneous distribution of 10000 emitters in a disk of radius $25 \mathrm{Mpc}$ and height $5 \mathrm{Mpc}$, centered on the Virgo cluster itself. The north pole of the supergalactic plane is at $l=47.37$ degrees, $b=+6.32$ degrees. The reference amplitude of each emitter will be $h_{\star}=10^{-17}$ or $h_{\star}=10^{-19}$ for EXPLORER and VIRGO respectively. According to recent work by Norris (2002) the faint, slowly-cooling Gamma Ray Bursts could be localized in the supergalactic plane. Although the effect is not yet confirmed it is interesting to predict what would be the rate of sources localized in the supergalactic plane.

5. Virgo and the possible Great Attractor. This distribution is made of 1000 emitters (galaxies) in the Virgo cluster, centered at the 1950-equatorial coordinates $B 122800.0+$ 124000 . We assumed a distance of $17 \mathrm{Mpc}$ and a radius of 1.7 Mpc. We expect that Virgo will produce the most easily detectable events (this is the reason why the Franco-Italian detector is called VIRGO). However, it has been suspected that a very huge structure, the Great-Attractor (Dressler et al. 1987; hereafter, GA), could be present at three times the distance of Virgo, but hidden by our Galaxy. From the acceleration induced on Virgo, one can assume that the GA has 27 more galaxies than Virgo. We thus added to our synthetic sample 27000 galaxies, centered at the 1950equatorial coordinates $B 150000.0-600000$. We assumed a distance of $51 \mathrm{Mpc}$ and a radius of 5.1 Mpc. For each emitter of both Virgo and the GA we assumed that the reference amplitude is $h_{\star}=10^{-17}$ or $h_{\star}=10^{-19}$ for EXPLORER and VIRGO respectively. With a high sensitivity detector, we expect that the GA could become the most efficient source of GW events. This could give a clue for the detection of this putative dense region.

\subsection{Practical method for constructing the samples}

The Cartesian $X, Y, Z$ coordinates of each emitter are chosen at random, for a uniform distribution, or following a Gaussian distribution around the center, for a discrete group like a cluster. These coordinates are chosen according to Fig. 1. The $X$ axis gives the direction of the origin of Right Ascension $(\gamma$ point). The North equatorial pole is defined by the $Z$-axis. The $X, Y, Z$ coordinates are then transformed into equatorial coordinates and distance. Then the coordinates are transformed into galactic or supergalactic coordinates, depending on the distribution we are constructing. An individual emitter is then accepted, or not, depending on its position with respect to the

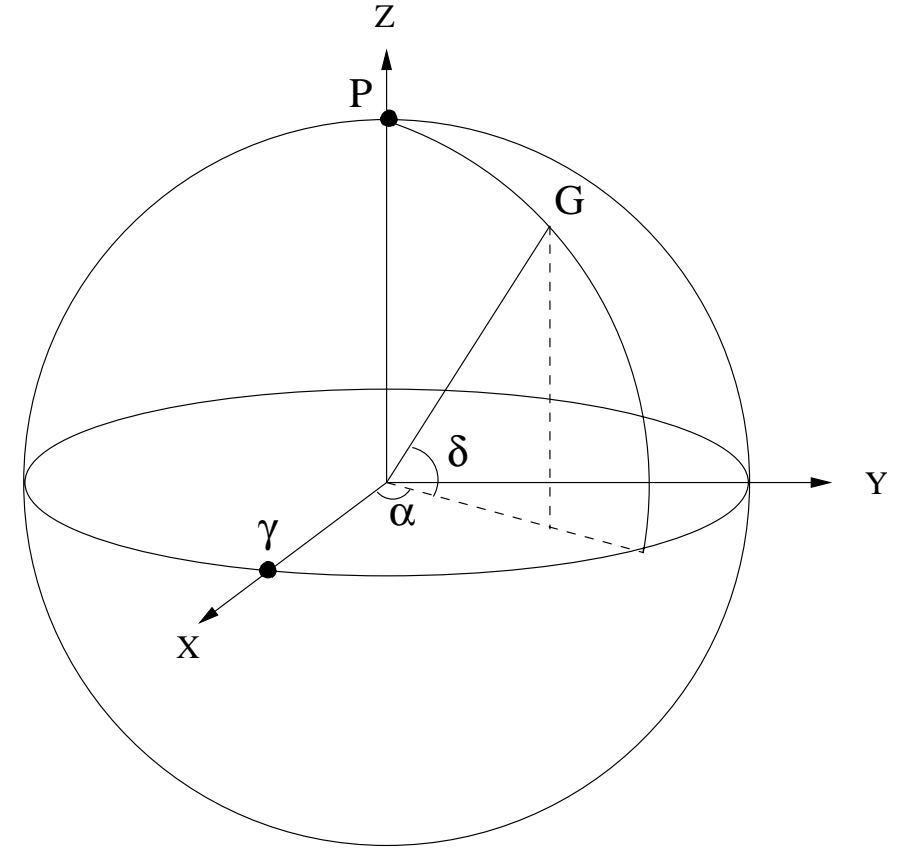

Fig. 1. Definition of the coordinates. The random $X, Y, Z$ coordinates define the position of an individual emitter $G$ (e.g., galaxy). The $X$-axis gives the origin of Right Ascension $\alpha$ ( $\gamma$ direction). The $X Y$-plane defines the equatorial plane used to define the declination $\delta$. Each random emitter $G$ is accepted (or rejected) for obeying the searched distribution.

distribution. This operation is repeated until the number of emitters has reached the desired value.

We considered the following detectors:

\begin{tabular}{llrl} 
Detector & Latitude $L$ & Azimuth $\Phi_{o}$ & Type \\
\hline EXPLORER & $46.5 \mathrm{deg}$ & $-39 \mathrm{deg}$ & bar \\
VIRGO & $43.6 \mathrm{deg}$ & $-19 \mathrm{deg}$ & interf. \\
\hline
\end{tabular}

The considered sensitivity range for EXPLORER is $10^{-18}$ to $10^{-20}$ and for VIRGO $10^{-20}$ to $10^{-22}$ The main useful characteristics are: the latitude on the Earth (let us recall that the longitude on the Earth does not intervene because the count rate of $\mathrm{GW}$ events is registered as a function of sidereal time), the azimuth of the main axis (the main axis of the bar or the $\mathrm{x}$-arm for an interferometric detector) and the type of detector.

\section{Results}

We calculated the GW normalized count rates as a function of sidereal time for the two detectors listed above. For each of the studied distributions we give a figure with three panels:

1. Panel 1 (top): a Flamsteed equal-area projection that shows the distribution in supergalactic coordinates.

2. Panel 2 (middle): the shape of the normalized count rate predicted for the EXPLORER bar detector along the sidereal time axis. We consider five sensitivity levels: $10^{-18.0}$, $10^{-18.5}, 10^{-19.0}, 10^{-19.5}$ and $10^{-20.0}$. The corresponding curves appear from the bottom to the top; the higher the sensitivity the higher the rate of events. 
3. Panel 3 (bottom): the shape of the normalized count rate predicted for the VIRGO interferometric detector along the sidereal time axis. We consider five sensitivity levels: $10^{-20.0}, 10^{-20.5}, 10^{-21.0}, 10^{-21.5}$ and $10^{-22.0}$. The corresponding curves can be identified as said for panel 2 .

The Figs. 2 to 6 give the results for respectively:

- a 3D homogeneous distribution of sources within $10 \mathrm{kpc}$;

- sources localized in the Galactic center;

- homogeneous distribution in the galactic plane;

- homogeneous distribution in the supergalactic plane;

- sources localized in the Virgo cluster and in the GreatAttractor.

Each curve gives the relative count number of GW events for equivalent sidereal time intervals centered at different sidereal times. The $Y$-axis is then a relative number of $\mathrm{GW}$ events per unit of time, i.e. a relative rate of events (in percents). We give an example: the middle panel of Fig. 4. It gives the rate of GW events for a homogeneous distribution of GW emitters in the galactic plane, as it would be seen from the EXPLORER bar detector. From the intermediate sensitivity $\left(10^{-18.5}\right)$ one can see that the rate of events detected around the sidereal time $S T=5 \mathrm{~h}(80 \%)$ is about twice the rate expected around $S T=21 \mathrm{~h}(35 \%)$. The corresponding points are marked with an open circle in Fig. 4. By comparing these kinds of curves with observed ones, it should be possible to have a signature of the localization of the GW sources. Then, from the sensitivity of the detector, one can place some limits on the radiated GW energy.

\section{Discussion and conclusions}

We have presented a method to analyze the output signal produced by GW detectors operating over a long time. This "Sidereal Time Analysis" method consists of recording all GW events as a function of sidereal time, in order to calculate their rate (number of events per unit of time) as a function of phase, from $0 \mathrm{~h}$ to $24 \mathrm{~h}$, for different sensitivity thresholds. If one admits that the noise is uniformly distributed along the sidereal time, the statistics of observed events can be performed even for very low signal to noise ratios (less than one).

A random distribution gives a flat count rate (Fig. 2). So, any sources distributed in such a homogeneous manner and close enough to be detected will appear as additional noise. Fortunately, the closest sources would have a galactic origin and would not lead to a flat count rate as shown by Figs. 3 and 4 . One can note the similarities between these two figures. The main characteristic of sources of tensor waves localized in our Galaxy (Galaxy center or Galaxy disk) is that their count rate shows a deep depression at $21 \mathrm{~h}$ sidereal time for the EXPLORER bar-detector and at $15 \mathrm{~h}$ and $22 \mathrm{~h}$ sidereal time for the VIRGO interferometric detector.

In Fig. 5 we see that the shape of the count rate for sources localized in the supergalactic plane shows almost opposite phase in comparison with Figs. 3 and 4. This is due to the fact that the supergalactic plane is almost perpendicular to the
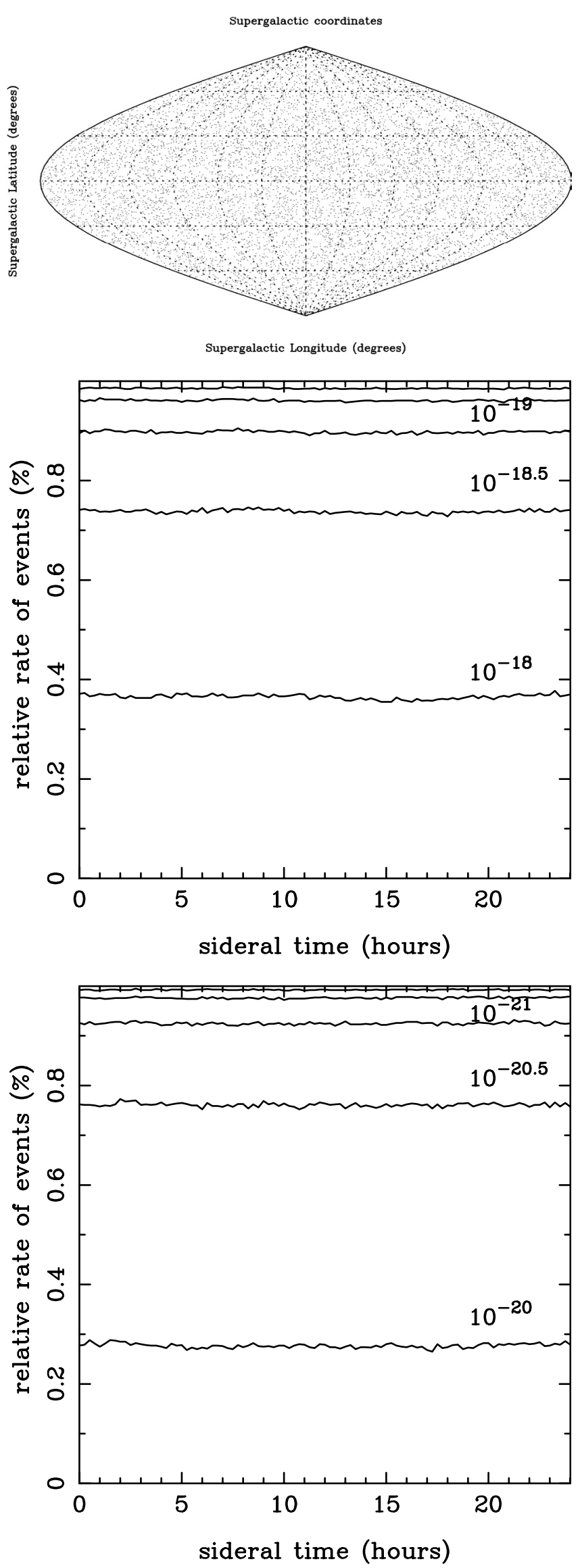

Fig. 2. 3D homogeneous distribution of sources within $10 \mathrm{kpc}$. See Sect. 3 for explanations. 

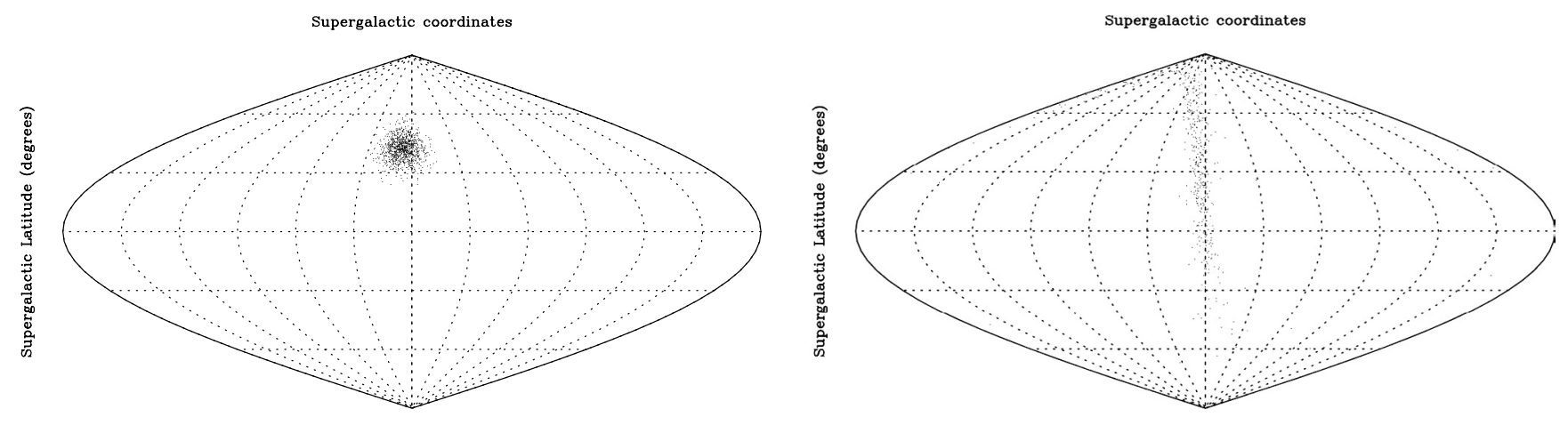

Supergalactic Longitude (degrees)
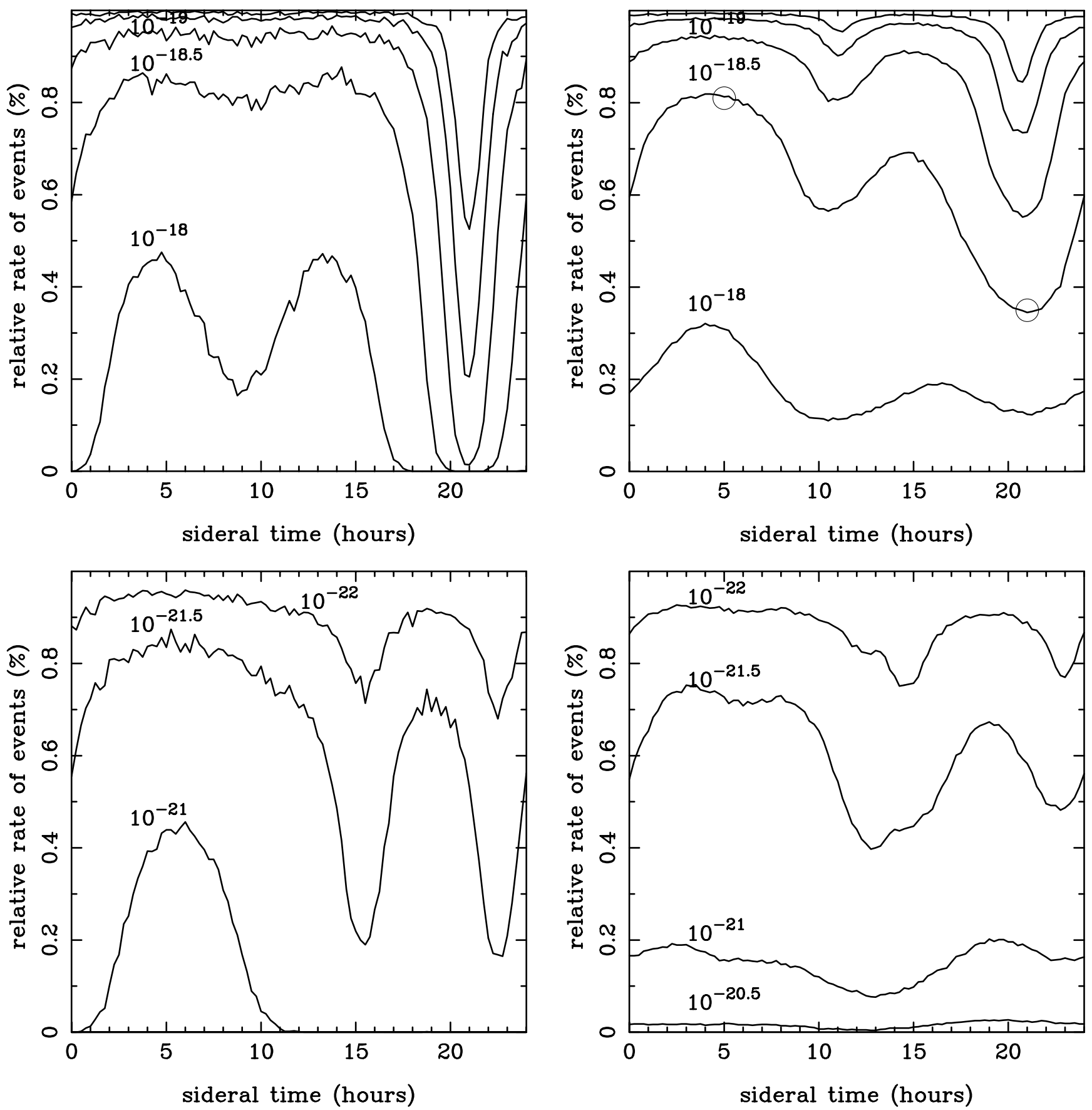

Fig. 3. Sources localized in the Galactic center. See Sect. 3 for explanations.

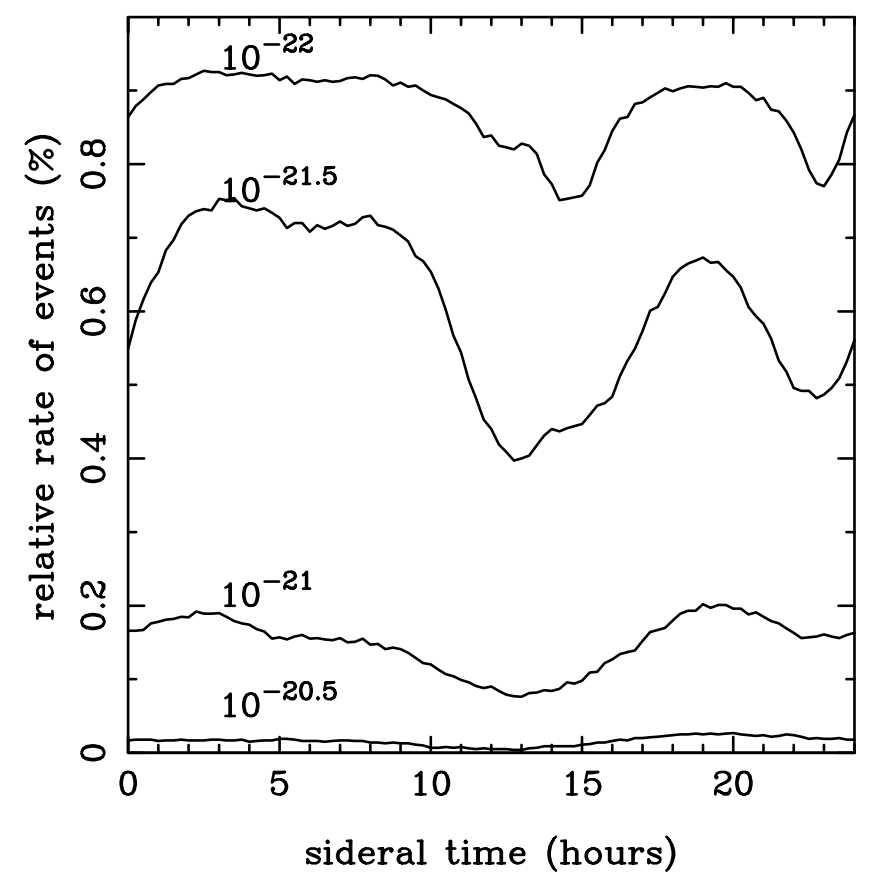

Fig. 4. Homogeneous distribution of sources in the galactic plane. See Sect. 3 for explanations. 

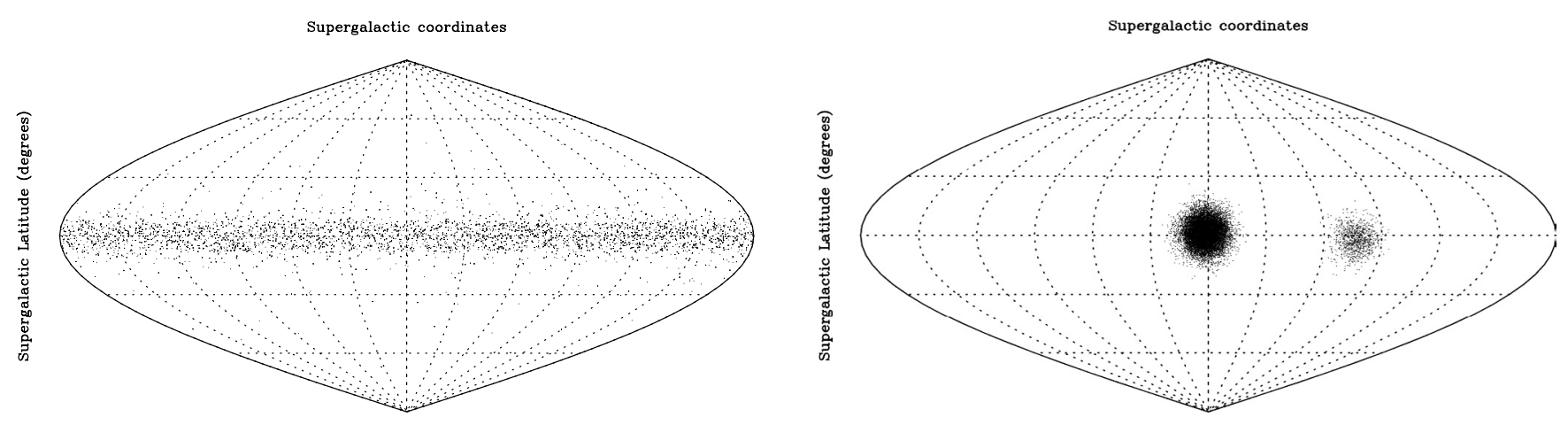

Supergalactic Longitude (degrees)
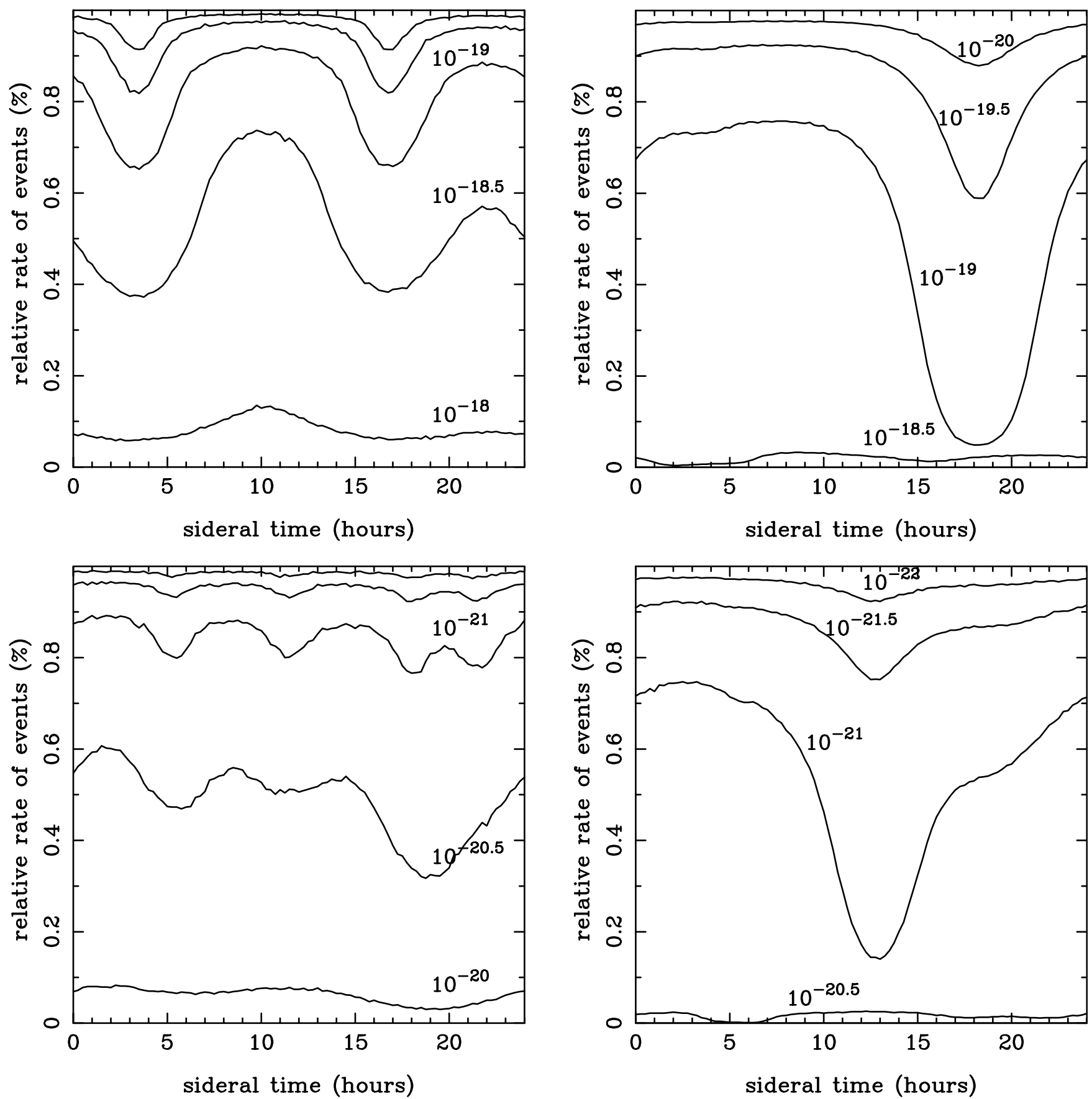

Fig. 5. Homogeneous distribution of sources in the supergalactic plane. See Sect. 3 for explanations.

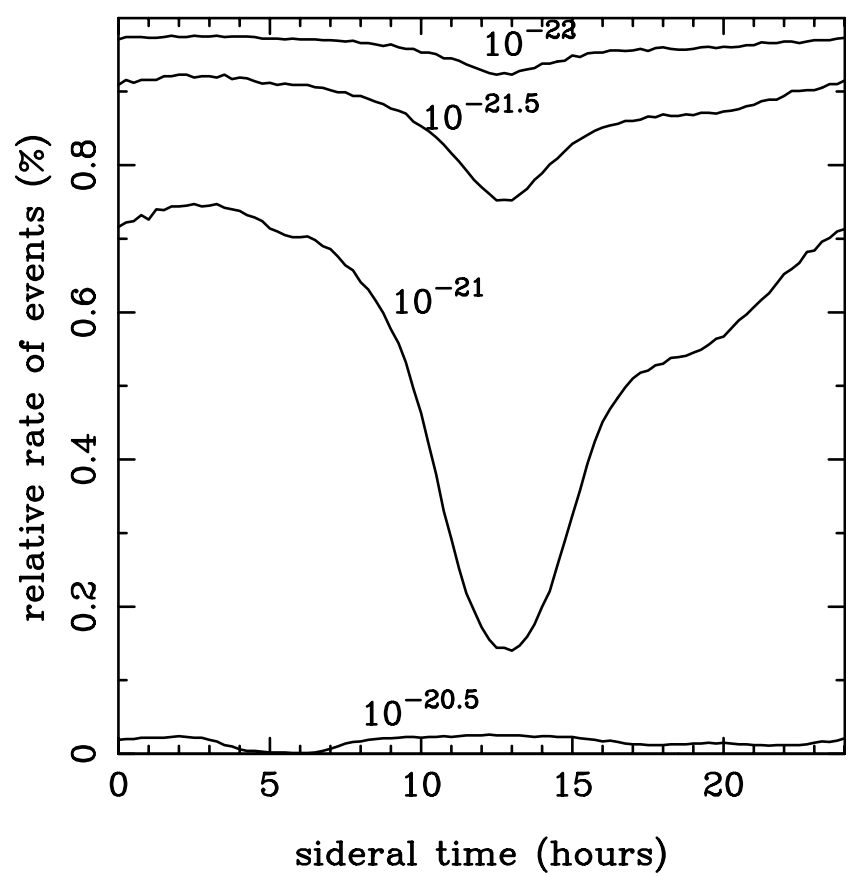

Fig. 6. Sources localized in the Virgo cluster and in the GreatAttractor. See Sect. 3 for explanations. 
galactic plane. This means that one can characterize the origin of a signal simply from the shape of its count rate.

Finally, the last calculations for the Virgo cluster and the putative GA also need comment (Fig. 6). We consider first the EXPLORER detector (middle panel). It shows that, at low sensitivity (lower curves), one will detect the Virgo cluster first, with a shape similar to the one found for the supergalactic plane (i.e., one peak at $10 \mathrm{~h}$ sidereal time and one peak at $23 \mathrm{~h}$ sidereal time) and that progressively one will detect a dominant region (between $2 \mathrm{~h}$ and $12 \mathrm{~h}$ with a big depression at $18 \mathrm{~h}$ ) when the GA dominates. So, when the sensitivity of the detectors are improved, it will be possible to test the existence of the GA.

For the VIRGO detector (Fig. 6 bottom panel) the effect is similar. We see a depression at $5 \mathrm{~h}$ when Virgo dominates, and a depression at $13 \mathrm{~h}$ when the GA dominates.

The next step consists of using real signals to start such an analysis. In particular, it will be possible to investigate the important case of coincident detections from multiple detectors and the case where one has only some events, the critical question being at what number of events can one have a significant result about the distribution of GW sources.

Acknowledgements. We thank the anonymous referee for his/her report and for suggesting further extensions of the paper.

\section{References}

Amaldi, E., \& Pizzella, G. 1979, in Relativity, Quanta and Cosmology in the development of the scientific thought of Albert Einstein, Johnson Rep. Corp. (Acad. Press), 241

Astone, P. 2001, in Proc. of 4th Amaldi Conference, Perth, July, 2001

Baryshev, Yu. V., \& Paturel, G. 2001, A\&A, 371, 378 (BP2001)

de Vaucouleurs 1956, Vistas Astron., 2, 1584

de Vaucouleurs 1975, ApJ, 202, 610

Dressler, A., Faber, S. M., Burstein, D., et al. 1987, ApJ, 313, L37

Finn, L. S. 2001, AIP Conf. Proc., 575, 92 [gr-qc/0104042]

Norris, J. 2002, ApJ, submitted [astro-ph/0201503]

Paczynski, B. 1999, in Proceedings of the STScI, Supernovae and Gamma Ray Bursts; the largest explosions since the Big Ban, ed. M. Livio, N. Pnagia, \& K. Sahu (Cambridge University Press), 1 [astro-ph/9909048]

Pizzella, G. 1989, in Gravitational Wave Data Analysis ed. B. F. Schutz (Kluwer Acad. Publishers), 173

Prodi, G., Heng, I. S., Allen, Z. A., et al. 2000, in Proc. the GWDAW99

Thorn, K. S. 1987, in Three Hundred Years of Gravitation

Thorn, K. S. 1997, in Proceeding of a Conference in Memory of S. Chandrasekhar, Black Holes and Relativistics Star, ed. R. M. Wald (University of Chicago Press, Chicago) [gr-qc/9706079]

Weber, J. 1969, Phys. Rev. Lett., 22, 1320 